PROCEEDINGS OF THE

AMERICAN MATHEMATICAL SOCIETY

Volume 134, Number 4, Pages 995-1001

S 0002-9939(05)08270-5

Article electronically published on October 7, 2005

\title{
ON RANKIN-COHEN BRACKETS FOR SIEGEL MODULAR FORMS
}

\author{
ÖZLEM IMAMOGLU AND OLAV K. RICHTER
}

(Communicated by David E. Rohrlich)

\begin{abstract}
We determine an explicit formula for a Rankin-Cohen bracket for Siegel modular forms of degree $n$ on a certain subgroup of the symplectic group. Moreover, we lift that bracket via a Poincaré series to a Siegel cusp form on the full symplectic group.
\end{abstract}

\section{INTRODUCTION}

In 1956, Rankin [10] showed that certain polynomials in the derivatives of modular forms are again modular forms. In 1977, Cohen [6] defined for each $\nu \geq 0$ an operator which assigns to two modular forms $f$ and $g$ of weight $k$ and $l$ a modular form $[f, g]_{\nu}$ of weight $k+l+2 \nu$. This operator is known as the Rankin-Cohen bracket, and operators similar in nature are called Rankin-Cohen-type brackets.

Explicit formulas for Rankin-Cohen brackets have been found for Jacobi forms ([1] and [2]), Siegel modular forms of degree 2 ([3] and [4]), and for Jacobi forms (of higher degree) on $\mathbb{H} \times \mathbb{C}^{n}([5])$. Eholzer and Ibukiyama [7] prove that there exists a unique Rankin-Cohen bracket for Siegel modular forms of arbitrary degree $n$. A closed formula for the Rankin-Cohen bracket is known only for $n=1$ and 2, even though a system of recursion relations is given for any degree $n$ in 7 .

In this paper, we consider Fourier-Jacobi expansions of Siegel modular forms. We demonstrate how a Rankin-Cohen bracket for Jacobi forms on $\mathbb{H} \times \mathbb{C}^{n-1}$ can be lifted to a Rankin-Cohen bracket for Siegel modular forms of degree $n$. In particular, for a certain subgroup of the symplectic group, we determine a closed formula for the Rankin-Cohen bracket. When $n=2$, that formula holds for the full symplectic group and our result coincides with Theorem 1.4 in [4, but if $n>2$, then our formula is valid only for the subgroup. However, in Theorem 3, we use our result to define a Poincaré series on the full symplectic group, which yields (for any $\nu \geq 0$ ) an operator that sends two Siegel modular forms (degree $n$ ) of weight $k_{1}$ and $k_{2}$ and a modular cusp form (degree 1) of weight $k_{3}$ to a Siegel cusp form (degree $n$ ) of weight $k_{1}+k_{2}+k_{3}+2 \nu$.

Received by the editors November 8, 2004 .

2000 Mathematics Subject Classification. Primary 11F46; Secondary 11F50, 11F60.

The first author was partially supported by the NSF. 


\section{JACOBI FORMS OF HIGHER DEGREE}

Let $\mathbb{A}$ be a commutative ring with unity and let $M_{m, n}(\mathbb{A})$ be the set of $m \times n$ matrices with entries in $\mathbb{A}$. If $U \in M_{n, n}(\mathbb{A})$, let $\operatorname{tr}(U)$ be the trace of $U$ and let $|U|$ be the determinant of $U$. We denote the symplectic group over the integers of degree $m$ by $\Gamma_{m}=\operatorname{Sp}_{m}(\mathbb{Z})$. Let $\Gamma_{m, j}$ be the subgroup of $\Gamma_{m}$ that consists of matrices $\left(\begin{array}{cc}A & B \\ C & D\end{array}\right)$ where $A=\left(\begin{array}{cc}A_{1} & 0 \\ A_{3} & A_{4}\end{array}\right), B=\left(\begin{array}{cc}B_{1} & B_{2} \\ B_{3} & B_{4}\end{array}\right), C=\left(\begin{array}{cc}C_{1} & 0 \\ 0 & 0\end{array}\right)$, and $D=\left(\begin{array}{cc}D_{1} & D_{2} \\ 0 & D_{4}\end{array}\right)$, where $A_{1}, B_{1}, C_{1}, D_{1} \in M_{j, j}(\mathbb{Z}), B_{2}, D_{2} \in M_{j, m-j}(\mathbb{Z}), A_{3}, B_{3} \in M_{m-j, j}(\mathbb{Z})$, and $A_{4}, B_{4}, D_{4} \in M_{m-j, m-j}(\mathbb{Z})$. The subgroup $\Gamma_{m, j}$ plays an important role in the theory of Siegel modular forms. For more details, see Freitag [9], chapter I, $\S 5$ and chapter II, §2. Let $\mathbb{H}_{m}$ be the Siegel upper half plane of degree $m, G: \mathbb{H}_{m} \rightarrow \mathbb{C}$, and let $\Gamma$ be a subgroup of $\Gamma_{m}$. As usual, for $M=\left(\begin{array}{cc}A & B \\ C & D\end{array}\right) \in \Gamma$ and for a positive integer $k$, we define the slash operator

$$
\left.G\right|_{k} M=G\left((A Z+B)(C Z+D)^{-1}\right)|C Z+D|^{-k}
$$

Let $F$ be a Siegel modular form of weight $k$ and degree $j+n-1$ on $\Gamma_{j+n-1}$, i.e., $F$ is holomorphic and $\left.F\right|_{k} M=F$ for all $M \in \Gamma_{j+n-1}$. We write $Z \in \mathbb{H}_{j+n-1}$ as $Z=\left(\begin{array}{cc}\tau & z \\ t_{z} & W\end{array}\right)$, where $\tau \in \mathbb{H}_{j}, z \in M_{j, n-1}(\mathbb{C})$, and $W \in \mathbb{H}_{n-1}$. The Fourier-Jacobi expansion of $F$ is given by

$$
F(Z)=F(\tau, z, W)=\sum_{\substack{\mathcal{M}={ }^{t} \mathcal{M} \geq 0 \\ \mathcal{M} \text { even }}} \Phi_{\mathcal{M}}(\tau, z) e^{\pi i t r(\mathcal{M} W)},
$$

where the sum is over symmetric, semi-positive definite, integral, and even $(n-1) \times(n-1)$ matrices $\mathcal{M}$. Note that $\Phi_{\mathcal{M}}$ is a Jacobi form of weight $k$ and index $\mathcal{M}$ in the sense of Ziegler [11, and that if $j=1$ and $n=2$, then $\Phi_{\mathcal{M}}$ is a Jacobi form in the sense of Eichler and Zagier [8]. Of particular interest is the case where $j=1$ and $n \geq 2$ is arbitrary. We denote the vector space of such Jacobi forms by $\mathcal{J}_{k, \mathcal{M}}\left(\Gamma_{1}\right)$.

Choie and Kim 5 provide an explicit formula for the Rankin-Cohen bracket for Jacobi forms on $\mathcal{J}_{k, \mathcal{M}}\left(\Gamma_{1}\right)$. We need the following definition to state their main result.

Definition 1. Let $\tau \in \mathbb{H}_{1}$ and $z=\left(z_{1}, \ldots, z_{n-1}\right) \in \mathbb{C}^{n-1}$. Suppose $\mathcal{M}=\left(m_{s t}\right)$ is a symmetric, positive definite, integral, and even $(n-1) \times(n-1)$ matrix, where $\mathcal{M}_{s t}$ is the cofactor of the entry $m_{s t}$. The heat operator $L_{\mathcal{M}}$ is defined by

$$
L_{\mathcal{M}}=4 \pi i|\mathcal{M}| \partial_{\tau}-\sum_{1 \leq s, t \leq n-1} \mathcal{M}_{s t} \partial_{z_{s}} \partial_{z_{t}}
$$

where $\partial_{x}=\frac{\partial}{\partial x}$.

Theorem 1 (Choie, Kim). Let $\phi_{1} \in \mathcal{J}_{k_{1}, \mathcal{M}_{1}}\left(\Gamma_{1}\right)$ and $\phi_{2} \in \mathcal{J}_{k_{2}, \mathcal{M}_{2}}\left(\Gamma_{1}\right)$. For each nonnegative integer $\nu$ and $Y \in \mathbb{C}$, set

$$
\begin{aligned}
& {\left[\left[\phi_{1}, \phi_{2}\right]\right]_{Y, \nu}} \\
& =\sum_{r+s+p=\nu} C_{r, s, p}\left(k_{1}, k_{2}\right) D_{r, s}\left(\mathcal{M}_{1}, \mathcal{M}_{2}, Y\right) L_{\mathcal{M}_{1}+\mathcal{M}_{2}}^{p}\left(L_{\mathcal{M}_{1}}^{r}\left(\phi_{1}\right) L_{\mathcal{M}_{2}}^{s}\left(\phi_{2}\right)\right),
\end{aligned}
$$

where

$$
C_{r, s, p}\left(k_{1}, k_{2}\right)=(-1)^{p} \frac{(\gamma+2 \nu-p-2) !}{r ! s ! p !(\alpha+r-1) !(\beta+s-1) !(\gamma+2 \nu-2) !},
$$




$$
\begin{aligned}
& D_{r, s}\left(\mathcal{M}_{1}, \mathcal{M}_{2}, Y\right) \\
& =\left(\frac{\left|\mathcal{M}_{1}+\mathcal{M}_{2}\right|}{\left|\mathcal{M}_{1}\right|+\left|\mathcal{M}_{2}\right|}+\left|\mathcal{M}_{2}\right| Y\right)^{r}\left(\frac{\left|\mathcal{M}_{1}+\mathcal{M}_{2}\right|}{\left|\mathcal{M}_{1}\right|+\left|\mathcal{M}_{2}\right|}-\left|\mathcal{M}_{1}\right| Y\right)^{s},
\end{aligned}
$$

and where $\alpha=k_{1}-\frac{n-1}{2}, \beta=k_{2}-\frac{n-1}{2}$, and $\gamma=k_{1}+k_{2}-\frac{n-1}{2}$. Then, $\left[\left[\phi_{1}, \phi_{2}\right]\right]_{Y, \nu} \in$ $\mathcal{J}_{k_{1}+k_{2}+2 \nu, \mathcal{M}_{1}+\mathcal{M}_{2}}\left(\Gamma_{1}\right)$.

Remark. Choie and Kim [5] also construct bilinear operators of the form $\mathcal{J}_{k_{1}, \mathcal{M}_{1}}\left(\Gamma_{1}\right)$ $\times \mathcal{J}_{k_{2}, \mathcal{M}_{2}}\left(\Gamma_{1}\right) \rightarrow \mathcal{J}_{k_{1}+k_{2}+2 \nu+1}, \mathcal{M}_{1}+\mathcal{M}_{2}\left(\Gamma_{1}\right)$, and they determine multilinear operators on $\mathcal{J}_{k, \mathcal{M}}\left(\Gamma_{1}\right)$.

\section{Rankin-COHEn type Brackets for Siegel Modular forms}

We will demonstrate how Theorem 1 can be used to construct an explicit formula for Rankin-Cohen brackets of holomorphic functions $F: \mathbb{H}_{n} \rightarrow \mathbb{C}$ that satisfy $\left.F\right|_{k} M=F$ for all $M \in \Gamma_{n, 1}$ for arbitrary $n \geq 2$.

As before, $Z=\left(\begin{array}{cc}\tau & z \\ t_{z} & W\end{array}\right) \in \mathbb{H}_{n}$, where $\tau \in \mathbb{H}_{1}, z=\left(z_{1}, \ldots, z_{n-1}\right) \in \mathbb{C}^{n-1}$, and $W=\left(w_{i j}\right) \in \mathbb{H}_{n-1}$. Set

$$
\mathbb{D}=\left|\begin{array}{cc}
2 \partial_{\tau} & \partial_{z} \\
{ }^{t} \partial_{z} & \partial_{W}
\end{array}\right|
$$

where $\partial_{z}=\left(\partial z_{1}, \ldots, \partial z_{n-1}\right)$ and $\partial_{W}=\left(1+\delta_{i j}\right) \frac{\partial}{\partial w_{i j}}$. Furthermore, let $\left|\partial_{W}\right|^{-1}$ be the inverse to the operator $\left|\partial_{W}\right|$ on the space of analytic functions of the form

$$
G(W)=\sum_{\substack{\mathcal{M}={ }^{t} \mathcal{M}>0 \\ \mathcal{M} \text { even }}} a(\mathcal{M}) e^{\pi i \operatorname{tr}(\mathcal{M} W)}
$$

More precisely, if $l \in \mathbb{Z}$, then

$$
\left|\partial_{W}\right|^{l} G(W)=(2 \pi i)^{(n-1) l} \sum_{\substack{\mathcal{M}={ }^{t} \mathcal{M}>0 \\ \mathcal{M} \text { even }}}|\mathcal{M}|^{l} a(\mathcal{M}) e^{\pi i \operatorname{tr}(\mathcal{M} W)} .
$$

Note that $\left|\partial_{W}\right|\left|\partial_{W}\right|^{-1} G(W)=\left|\partial_{W}\right|^{-1}\left|\partial_{W}\right| G(W)=G(W)$. We have the following theorem.

Theorem 2. Let $F_{l}: \mathbb{H}_{n} \rightarrow \mathbb{C}$ be holomorphic such that $\left.F_{l}\right|_{k_{l}} M=F_{l}$ for all $M \in \Gamma_{n, 1}$ where $l=1,2$. For each nonnegative integer $\nu$, set

$$
F=\left[F_{1}, F_{2}\right]_{\nu}
$$

$$
=\sum_{r+s+p=\nu} C_{r, s, p}\left(k_{1}, k_{2}\right) \mathbb{D}^{p}\left(\left|\partial_{W}\right|^{-p} \sum_{l=0}^{p}\left(\begin{array}{c}
p \\
l
\end{array}\right) \mathbb{D}^{r}\left(\left|\partial_{W}\right|^{l} F_{1}\right) \mathbb{D}^{s}\left(\left|\partial_{W}\right|^{p-l} F_{2}\right)\right),
$$

where $C_{r, s, p}\left(k_{1}, k_{2}\right)$ is as in Theorem 1, Then $\left.F\right|_{k_{1}+k_{2}+2 \nu} M=F$ for all $M \in \Gamma_{n, 1}$. Moreover, if $\nu>0$, then $F$ is in the kernel of the Siegel $\phi$-operator.

Proof. We begin by noting that

$$
\sum_{l=0}^{p}\left(\begin{array}{c}
p \\
l
\end{array}\right) \mathbb{D}^{r}\left(\left|\partial_{W}\right|^{l} F_{1}(Z)\right) \mathbb{D}^{s}\left(\left|\partial_{W}\right|^{p-l} F_{2}(Z)\right)
$$

has a Fourier series as in (44). Hence applying $\left|\partial_{W}\right|^{-p}$ in (6) is well defined. If $\nu=0$, then $\left[F_{1}, F_{2}\right]_{0}=F_{1} F_{2}$. From now on we assume that $\nu>0$. For $l=1,2$ let

$$
F_{l}(Z)=\sum_{\substack{T={ }^{t} T \geq 0 \\ T \text { even }}} a_{l}(T) e^{\pi i \operatorname{tr}(T Z)}=\sum_{\substack{\mathcal{M}={ }^{t} \mathcal{M} \geq 0 \\ \mathcal{M} \text { even }}} \Phi_{\mathcal{M}}^{(l)}(\tau, z) e^{\pi i t r(\mathcal{M} W)}
$$


It is crucial to realize that if $\phi: \mathbb{H} \times \mathbb{C}^{n-1} \rightarrow \mathbb{C}$ is analytic, then

$$
\mathbb{D}\left(\phi e^{\pi i \operatorname{tr}(\mathcal{M} W)}\right)=(2 \pi i)^{n-2} L_{\mathcal{M}}(\phi) e^{\pi i \operatorname{tr}(\mathcal{M} W)},
$$

where $L_{\mathcal{M}}$ is the heat operator in (21). The key step in our proof is to use (7) to express $F=\left[F_{1}, F_{2}\right]_{\nu}$ as

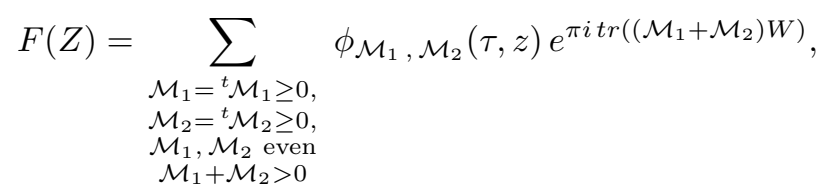

where

$$
\phi_{\mathcal{M}_{1}, \mathcal{M}_{2}}(\tau, z)=(2 \pi i)^{(n-2) \nu}\left(\frac{\left|\mathcal{M}_{1}\right|+\left|\mathcal{M}_{2}\right|}{\left|\mathcal{M}_{1}+\mathcal{M}_{2}\right|}\right)^{\nu}\left[\left[\phi_{\mathcal{M}_{1}}^{(1)}(\tau, z), \phi_{\mathcal{M}_{2}}^{(2)}(\tau, z)\right]\right]_{0, \nu}
$$

To verify that $F(Z)$ is indeed a modular form of weight $k_{1}+k_{2}+2 \nu$ on $\Gamma_{n, 1}$, it suffices to check the behavior under modular transformations for a set of generators of $\Gamma_{n, 1}$ : For $\gamma=\left(\begin{array}{ll}a & b \\ c & d\end{array}\right) \in \mathrm{SL}_{2}(\mathbb{Z})$, set

$$
\bar{\gamma}=\left(\begin{array}{ll}
A & B \\
C & D
\end{array}\right)=\left(\begin{array}{cccc}
a & 0 & b & 0 \\
0 & I_{n-1} & 0 & 0 \\
c & 0 & d & 0 \\
0 & 0 & 0 & I_{n-1}
\end{array}\right) \in \Gamma_{n, 1}
$$

and for $\lambda=\left(\lambda_{1}, \ldots, \lambda_{n-1}\right), \mu=\left(\mu_{1}, \ldots, \mu_{n-1}\right) \in \mathbb{Z}^{n-1}$ set

$$
\left(\begin{array}{ll}
A^{\prime} & B^{\prime} \\
C^{\prime} & D^{\prime}
\end{array}\right)=\left(\begin{array}{cccc}
1 & 0 & 0 & \mu \\
{ }^{t} \lambda & I_{n-1} & { }^{t} \mu & 0 \\
0 & 0 & 1 & -\lambda \\
0 & 0 & 0 & I_{n-1}
\end{array}\right) \in \Gamma_{n, 1} .
$$

By Theorem 1, $\left[\left[\phi_{\mathcal{M}_{1}}^{(1)}(\tau, z), \phi_{\mathcal{M}_{2}}^{(2)}(\tau, z)\right]\right]_{0, \nu} \in \mathcal{J}_{k_{1}+k_{2}+2 \nu, \mathcal{M}_{1}+\mathcal{M}_{2}}\left(\Gamma_{1}\right)$. This implies that $\left.F\right|_{k_{1}+k_{2}+2 \nu}\left(\begin{array}{ll}A & B \\ C & D\end{array}\right)=F$ and also that $\left.F\right|_{k_{1}+k_{2}+2 \nu}\left(\begin{array}{cc}A^{\prime} & B^{\prime} \\ C^{\prime} & D^{\prime}\end{array}\right)=F$.

Observe that $\Gamma_{n}$ is generated by

$$
\left\{\left(\begin{array}{cc}
{ }^{t} X & { }^{t} X S \\
0 & X^{-1}
\end{array}\right) \mid X \in \mathrm{GL}_{n}(\mathbb{Z}), S={ }^{t} S \in \mathrm{M}_{n, n}(\mathbb{Z})\right\} \cup\left\{\bar{\gamma} \mid \gamma \in \mathrm{SL}_{2}(\mathbb{Z})\right\}
$$

and that $\mathrm{GL}_{n}(\mathbb{Z})$ is generated by matrices of the following type:

1. $\left\{\left(1+r \delta_{i j}\right) I_{n} \mid 1 \leq i<j \leq n, r \in \mathbb{Z}\right\}$.

2. $\left\{P_{\sigma}=\left(\delta_{j \sigma(j)}\right) \mid \sigma \in \mathbb{S}_{n}\right\}$.

3. $\left\{\left({ }^{\epsilon} I_{n-1}\right) \mid \epsilon= \pm 1\right\}$.

One can check that $\Gamma_{n, 1}$ is generated by

$$
\left\{\left(\begin{array}{cc}
{ }^{t} X & { }^{t} X S \\
0 & X^{-1}
\end{array}\right) \mid X \in \mathrm{GL}_{n}^{\prime}(\mathbb{Z}), S={ }^{t} S \in \mathrm{M}_{n, n}(\mathbb{Z})\right\} \cup\left\{\bar{\gamma} \mid \gamma \in \mathrm{SL}_{2}(\mathbb{Z})\right\},
$$

where $\mathrm{GL}_{n}^{\prime}(\mathbb{Z})$ is generated by matrices of the following type:

1. $\left\{\left(1+r \delta_{i j}\right) I_{n} \mid 1 \leq i<j \leq n, r \in \mathbb{Z}\right\}$.

$2^{\prime} .\left\{P_{\sigma}=\left(\delta_{j \sigma(j)}\right) \mid \sigma \in \mathbb{S}_{n}, \sigma(1)=1\right\}$.

3. $\left\{\left(\begin{array}{c}{ }^{\epsilon} I_{n-1} \\ I\end{array}\right) \mid \epsilon= \pm 1\right\}$. 
A straightforward computation reveals that

$$
F(Z)=(2 \pi i)^{n \nu} \sum_{r+s+p=\nu} C_{r, s, p}\left(k_{1}, k_{2}\right) \sum_{\substack{T={ }^{t} T>0 \\ T \text { even }}} C_{r, s, p}(T) e^{\pi i \operatorname{tr}(T Z)},
$$

where

$$
C_{r, s, p}(T)=\sum_{\substack{T_{1}+T_{2}=T, T_{1}={ }^{t} T_{1} \geq 0, T_{2}={ }^{t} T_{2} \geq 0, T_{1}, T_{2} \text { even }}} a_{1}\left(T_{1}\right) a_{2}\left(T_{2}\right)\left|T_{1}\right|^{r}\left|T_{2}\right|^{s}\left|T_{1}+T_{2}\right|^{p}\left(\frac{\left|T_{1}^{*}\right|+\left|T_{2}^{*}\right|}{\left|T_{1}^{*}+T_{2}^{*}\right|}\right)^{p},
$$

and where $T_{l}=\left(\begin{array}{l}* \\ *\end{array} T_{l}^{*}\right)$ with $T_{l}^{*}={ }^{t} T_{l}^{*} \in M_{n-1, n-1}(\mathbb{Z})$. Consequently, $F(Z+S)=$ $F(Z)$ for all symmetric $S \in \mathrm{M}_{n, n}(\mathbb{Z})$.

Note that if $X$ is a generator of $\mathrm{GL}_{n}^{\prime}(\mathbb{Z})$, then $\left|\left(X^{-1} T^{t} X^{-1}\right)^{*}\right|=\left|T^{*}\right|$ for all symmetric $T \in M_{n, n}(\mathbb{Z})$. Since

$$
a_{1}\left(X^{-1} T_{1}{ }^{t} X^{-1}\right) a_{2}\left(X^{-1} T_{2}{ }^{t} X^{-1}\right)=|X|^{k_{1}+k_{2}} a_{1}\left(T_{1}\right) a_{2}\left(T_{2}\right),
$$

we find that $C_{r, s, p}\left(X^{-1} T^{t} X^{-1}\right)=|X|^{k_{1}+k_{2}} C_{r, s, p}(T)$. Hence by equation (11),

$$
F\left({ }^{t} X Z X\right)=|X|^{k_{1}+k_{2}+2 \nu} F(Z) .
$$

We conclude that $\left.F\right|_{k_{1}+k_{2}+2 \nu} M=F$ for all $M \in \Gamma_{n, 1}$. Moreover, equation (11) shows that $F(Z)$ is in the kernel of the $\phi$-operator.

Remarks. 1) If $n=2$, then $\frac{\left|T_{1}^{*}\right|+\left|T_{2}^{*}\right|}{\left|T_{1}^{*}+T_{2}^{*}\right|}=1$ in equation (11), which yields that equation (12) is valid for all $X \in \mathrm{GL}_{2}(\mathbb{Z})$. Hence $\left.F\right|_{k_{1}+k_{2}+2 \nu} M=F$ for all $M \in \Gamma_{2}$, $F$ differs only by a constant from the Rankin-Cohen bracket in [4, and Theorem 2 reduces to Theorem 1.4 of 4 .

2) If $n>2$, then $\left[F_{1}, F_{2}\right]_{\nu}$ is not necessarily a Siegel modular form on the full symplectic group $\Gamma_{n}$. For example, if $F_{1}=F_{2}$ is the theta function associated to an even unimodular lattice, then $\left[F_{1}, F_{2}\right]_{\nu}$ is a theta function with polynomial coefficients. However, equation (11) implies that the polynomial is not harmonic, and hence $\left[F_{1}, F_{2}\right]_{\nu}$ is not a Siegel modular form on $\Gamma_{n}$.

3) Choie and Kim [5] also determine multilinear operators on $\mathcal{J}_{k, \mathcal{M}}\left(\Gamma_{1}\right)$. One can use their result to construct multilinear operators on Siegel modular forms of degree $n$. This allows one to generalize the main result in Choie [3] to the case where $n>2$.

Next we proceed as in Freitag 9 to lift $\left[F_{1}, F_{2}\right]_{\nu}$ to a Siegel modular form on $\Gamma_{n}$. If $Z=X+i Y \in \mathbb{H}_{n}$, then let $z_{1}$ and $y_{1}$ be the (1,1)-entries of $Z$ and $Y$, respectively.

Theorem 3. Let $F_{l}$ be Siegel modular forms of weight $k_{l}$ and degree $n$ on $\Gamma_{n}$ for $l=1,2$, and let $f_{3}$ be a modular cusp form of weight $k_{3}$ and degree 1 on $\Gamma_{1}$. For each nonnegative integer $\nu$, set $F(Z)=\left[F_{1}, F_{2}\right]_{\nu}$ and $F_{3}(Z)=f_{3}\left(z_{1}\right)$. If $k_{1}+k_{2}+k_{3}+2 \nu>2 n^{2}-n+6$, then

$$
P_{F_{1}, F_{2}, f_{3}}(Z)=\left.\sum_{M \in \Gamma_{n, 1} \backslash \Gamma_{n}}\left(F F_{3}\right)\right|_{k_{1}+k_{2}+k_{3}+2 \nu} M
$$

is a Siegel modular form of weight $k_{1}+k_{2}+k_{3}+2 \nu$ on $\Gamma_{n}$. Moreover, if $\nu>0$, then $P_{F_{1}, F_{2}, f_{3}}(Z)$ is a Siegel cusp form on $\Gamma_{n}$. 
Remarks. 1) If $n=2,\left.F\right|_{k_{1}+k_{2}+2 \nu} M=F$ for all $M \in \Gamma_{2}$ and $F$ differs only by a constant from the Rankin-Cohen bracket in [4]. Furthermore, $P_{F_{1}, F_{2}, f_{3}}(Z)=$ $F(Z) E_{f_{3}}(Z)$, where $E_{f_{3}}(Z)=\left.\sum_{M \in \Gamma_{2,1} \backslash \Gamma_{2}} F_{3}\right|_{k_{3}} M$ is the Klingen-Eisenstein series attached to the cusp form $f_{3}$. For $n>2$, as in the classical case of Poincare series, it seems difficult to determine when the series in equation (13) vanishes identically.

2 ) If $n>2$, then Theorem 3 does not coincide with the Rankin-Cohen bracket in [7. However, it gives a construction of a Siegel cusp form of weight $k_{1}+k_{2}+k_{3}+2 \nu$ attached to two Siegel cusp forms of weight $k_{1}$ and $k_{2}$ and an elliptic cusp form of weight $k_{3}$.

Proof. We have $\left.F\right|_{k_{1}+k_{2}+2 \nu} M=F$ and $\left.F_{3}\right|_{k_{3}} M=F_{3}$ for all $M \in \Gamma_{n, 1}$. Hence $P_{F_{1}, F_{2}, f_{3}}(Z)$ transforms like a Siegel modular form of weight $k=k_{1}+k_{2}+k_{3}+2 \nu$ on $\Gamma_{n}$. It remains to show that $P_{F_{1}, F_{2}, f_{3}}(Z)$ converges.

If $\nu=0$, then $P_{F_{1}, F_{2}, f_{3}}(Z)=F_{1}(Z) F_{2}(Z) E_{f_{3}}(Z)$ converges whenever $k_{3}>$ $n+2$ (see I. 5.4 in [9]). For $\nu>0$, set $G(Z)=y_{1}^{\left(\frac{k}{2}-N\right)}|Y|^{N}\|F(Z)\|$, where $(n-1)^{2}+1<N<k / 2$, and where $\|\cdot\|$ denotes the absolute value. We will show that $G(Z)$ is bounded on $\mathbb{H}_{n}$, which then yields the convergence of $P_{F_{1}, F_{2}, f_{3}}(Z)$. Note that $\left.G\right|_{k} M=G$ for all $M \in \Gamma_{n, 1}$. There exists $u>1$ such that $\mathcal{F}_{n, 1}[u]$ is a fundamental set for $\Gamma_{n, 1}$ (for details see I. $\S 5$ in [9]). Let $Z=X+i Y \in \overline{\mathcal{F}_{n, 1}[u]}$. Hence $Y=\left(\begin{array}{cc}1 & 0 \\ t_{b} & I_{n-1}\end{array}\right)\left(\begin{array}{cc}y_{1} & 0 \\ 0 & Y_{2}\end{array}\right)\left(\begin{array}{cc}1 & b \\ 0 & I_{n-1}\end{array}\right)$, where $y_{1} \geq \frac{1}{u}, b^{t} b \leq u$, and $Y_{2}={ }^{t} Y_{2}=$ $\left(\begin{array}{ccc}y_{22} & \cdots & y_{2 n} \\ \vdots & & \vdots \\ y_{2 n} & \cdots & y_{n n}\end{array}\right)>0$ with $y_{j j} \leq u y_{j+1} j+1(2 \leq j \leq n-1),\left\|y_{i j}\right\| \leq u y_{j j}(2 \leq i, j \leq n)$, and $y_{22} \cdots y_{n n} \leq u\left|Y_{2}\right|$.

We apply a standard estimate (Lemma 2.6 of [11]) to the Jacobi cusp form $\phi_{\mathcal{M}_{1}, \mathcal{M}_{2}}(\tau, z)$ in equation (8) and find

$$
G(Z) \leq C y_{1}^{\frac{k_{3}}{2}}\left\|f_{3}(\tau)\right\|\left|Y_{2}\right|^{N} \sum_{\substack{\mathcal{M}={ }^{t} \mathcal{M}>0 \\ \mathcal{M} \text { even }}} e^{-\pi \operatorname{tr}\left(\mathcal{M} Y_{2}\right)},
$$

for some $C>0$. The right-hand side of (14) clearly vanishes when $y_{1} \rightarrow \infty\left(f_{3}\right.$ is a cusp form) or when $y_{n n} \rightarrow \infty$. It remains to show that $G(Z)$ vanishes when $y_{22} \rightarrow 0$. Let $\tilde{Y}_{2}=\left(\begin{array}{ccc}y_{22} & & \\ & \ddots & \\ & & y_{n n}\end{array}\right)$. It is not difficult to see that there exists $\delta_{n}>0$ such that $\delta_{n} \tilde{Y}_{2} \leq Y_{2} \leq \delta_{n}{ }^{-1} \tilde{Y}_{2}$. Let

$$
\sigma(n, l)=\#\left\{\mathcal{M} \mid \mathcal{M} \in M_{n-1, n-1}(\mathbb{Z}),{ }^{t} \mathcal{M}=\mathcal{M}>0, \mathcal{M} \text { even }, \operatorname{tr}(\mathcal{M})=l\right\} .
$$

Then

$$
\begin{aligned}
\left|Y_{2}\right|^{N} & \sum_{\substack{\mathcal{M}=^{t} \mathcal{M}>0 \\
\mathcal{M} \text { even }}} e^{-\pi \operatorname{tr}\left(\mathcal{M} Y_{2}\right)} \\
\leq & \left(\delta_{n}^{-n} y_{22} \cdots y_{n n}\right)^{N} \sum_{\substack{\mathcal{M}={ }^{t} \mathcal{M}>0 \\
\mathcal{M} \text { even }}} e^{-\pi \delta_{n} y_{22} \operatorname{tr}(\mathcal{M})}, \\
& =\left(\delta_{n}^{-n} y_{22} \cdots y_{n n}\right)^{N} \sum_{l>0} \sigma(n, l) e^{-\pi \delta_{n} y_{22} l}, \\
& \leq\left(\delta_{n}^{-n} y_{22} \cdots y_{n n}\right)^{N} \sum_{l>0} l^{(n-1)^{2}}\left(e^{-\pi \delta_{n} y_{22}}\right)^{l}
\end{aligned}
$$


If $N>(n-1)^{2}+1$, then the right side vanishes when $y_{22} \rightarrow 0$. Hence $G(Z)$ is bounded on $\overline{\mathcal{F}_{n, 1}[u]}$ and consequently also on $\mathbb{H}_{n}$ (see also I. 3.11 in [9]).

Let $h(Z)=y_{1}^{-\left(\frac{k}{2}-N\right)}$ and $H(Z)=|Y|^{-N}$. Then

$$
E_{h H}(Z)=\sum_{M \in \Gamma_{n, 1} \backslash \Gamma_{n}}\left\|\left.(h H)\right|_{k} M\right\|=H(Z) \sum_{M \in \Gamma_{n, 1} \backslash \Gamma_{n}}\left\|\left.h\right|_{k-2 N} M\right\|
$$

converges absolutely (see I. 5.4 in [9]) if $k-2 N>n+2$. Hence $P_{F_{1}, F_{2}, f_{3}}(Z)$ converges if $k>2 n^{2}-n+6$. Finally, if $\nu>0$, then it is easy to check that $P_{F_{1}, F_{2}, f_{3}}(Z)$ is in the kernel of the $\phi$-operator (compare with p. 72 in [9]), i.e., $P_{F_{1}, F_{2}, f_{3}}(Z)$ is a Siegel cusp form.

\section{ACKNOWLEDGMENT}

The second author thanks William Cherry for useful discussions.

\section{REFERENCES}

1. Y. Choie, Jacobi forms and the heat operator, Math. Z. 225 (1997), 95-101. MR1451334 (98c:11042)

2. Jacobi forms and the heat operator II, Illinois J. Math. 42 (1998), no. 2, 179-186. MR:1612731 (99d:11049)

3. Multilinear operators on Siegel modular forms of genus 1 and 2, J. Math. Anal. Appl. 232 (1999), 34-44. MR.1683034 (2000a:11074)

4. Y. Choie and W. Eholzer, Rankin-Cohen operators for Jacobi and Siegel forms, J. Number Theory 68 (1998), 160-177. MR1605899 (99b:11050)

5. Y. Choie and H. Kim, Differential operators on Jacobi forms of several variables, J. Number Theory 82 (2000), 140-163. MR.1755161 (2001g:11069)

6. H. Cohen, Sums involving the values at negative integers of $L$-functions and quadratic characters, Math. Ann. 217 (1975), 271-285. MR0382192 (52:3080)

7. W. Eholzer and T. Ibukiyama, Rankin-Cohen type differential operators for Siegel modular forms, Internat. J. Math. 9 (1998), no. 4, 443-463. MR1635181(2000c:11079)

8. M. Eichler and D. Zagier, The theory of Jacobi forms, Birkhäuser, Boston, 1985. MR0781735 (86j:11043)

9. E. Freitag, Siegelsche Modulfunktionen, Springer, Berlin, Heidelberg, New York, 1983. MR.0871067 (88b:11027)

10. R. Rankin, The construction of automorphic forms from the derivatives of a given form, J. Indian Math. Soc. 20 (1956), 103-116. MR0082563 (18:571c)

11. C. Ziegler, Jacobi forms of higher degree, Abh. Math. Sem. Univ. Hamburg, 59 (1989), 191224. MR1049896 (91g:11046)

Department of Mathematics, Eidgenössische Technische Hochschule, CH-8092, ZÜRICH, SWITZERLAND

E-mail address: ozlem@math.ethz.ch

Department of Mathematics, University of North Texas, Denton, Texas 76203

E-mail address: richter@unt.edu 